\title{
Impacto femoroacetabular - Fatores associados à presença de lesões profundas da junção condrolabral*
}

\section{Femoroacetabular Impingement - Factors Associated with the Presence of Deep Injuries of the Chondrolabral Junction}

\author{
Samuel Faccioni ${ }^{1}$ Vinicius Adelchi Cachoeira ${ }^{1}$ Gabriel Pozzobon Knop ${ }^{1}$ \\ Luiz Henrique Penteado Silva ${ }^{1}$ Tercildo Knop ${ }^{1}$ \\ ${ }^{1}$ Instituto de Ortopedia e Traumatologia, Hospital São Vicente de \\ Paulo, Passo Fundo, RS, Brasil \\ Rev Bras Ortop 2019;54:434-439. \\ Endereço para correspondência Samuel Faccioni, Instituto de \\ Ortopedia e Traumatologia (IOT), Hospital São Vicente de Paulo, Passo \\ Fundo, RS, 99010-112, Brasil \\ (e-mail: faccionis@yahoo.com.br; pesquisa2@hsvp.com.br).
}

\section{Resumo \\ Palavras-chave \\ - impacto femoroacetabular \\ - artroscopia \\ - quadril}

\section{Abstract}

Objetivo Avaliar os fatores associados à presença de lesões condrais profundas (graus III e IV de Konan/Haddad) em pacientes submetidos à artroscopia do quadril para tratamento do impacto femoroacetabular (IFA).

Método Estudo transversal, prospectivo, de uma série de 125 artroscopias consecutivas do quadril feitas entre maio de 2016 e maio de 2017. Depois de aplicados os critérios de exclusão, foram analisados 107 quadris de 92 pacientes submetidos a tratamento cirúrgico do IFA dos tipos misto e CAM. Para fins de análise, os grupos foram divididos entre lesões consideradas leves e profundas, e foi feita associação com escore de sintomas, ângulo de cobertura lateral, ângulo alfa, idade, gênero, e classificação radiológica de artrose. Foram considerados como estatisticamente significativos testes com valor de probabilidade $<0,05$.

Resultados Pacientes cujos quadris apresentaram lesões consideradas profundas tiveram escores de quadril não artrítico (NAHSs, na sigla em inglês) significativamente maiores do que aqueles cujos quadris apresentavam lesões consideradas leves ou não apresentavam lesão condrolabral $(67,9 \pm 19,4$ versus $57,0 \pm 21,9 ; p=0,027)$. A prevalência de lesões profundas foi maior nos quadris Tonnis 1 do que nos que apresentaram Tonnis $0: 15$ (55,6\%) versus $10(12,7 \%)$, respectivamente; $p<0,001$. Homens apresentaram melhores escores funcionais e maior prevalência de lesões graus III e IV do que as mulheres: $65,6 \pm 19,6$ versus $49,3 \pm 21,6 ; p<0,001$, e 23 (34,3\%) versus $2(5,0 \%), p=0,001$, respectivamente.

Conclusão Homens apresentaram maior prevalência de lesões profundas. Quadris Tonnis 1 tiveram um risco 4,4 vezes maior de apresentar essas lesões. Pacientes com lesões condrolabrais profundas apresentaram melhor escore funcional pré-operatório.

Objective The purpose of the present study was to evaluate factors associated with the presence of deep chondral lesions (Konan/Haddad grades III and IV) in patients submitted to hip arthroscopy to treat femoroacetabular impingement (FAI).

Publicado originalmente por Elsevier Editora Ltda.

(D) Samuel Faccioni's ORCID is https://orcid.org/0000-0002-3991-1499.

recebido

24 de Agosto de 2017

aceito

03 de Abril de 2018
DOI https://doi.org/

10.1016/j.rbo.2018.04.003. ISSN 0102-3616.
Copyright $(2019$ by Sociedade Brasileira License terms de Ortopedia e Traumatologia. Published by Thieme Revnter Publicações Ltda, Rio de Janeiro, Brazil 
Method This was a prospective, cross-sectional study of a series of 125 consecutive hip arthroscopies performed between May 2016 and May 2017. After applying the exclusion criteria, 107 hips of 92 patients submitted to surgical treatment for mixed and CAM FAI were analyzed. For purposes of analysis, the present study considered groups with lesions considered mild and deep, which were associated with symptom score, lateral coverage angle, $\alpha$ angle, age, gender, and radiological classification of arthrosis. Results with a $p$-value $<0.05$ were considered statistically significant.

Results Patients whose hips had lesions considered deep had significantly higher nonarthritic hip scores (NAHSs) than those whose hips presented lesions considered mild or who did not present chondral lesions (67.9 \pm 19.4 versus $57.0 \pm 21.9, p=0.027)$. The prevalence of deep lesions was higher in hips with Tonnis 1 compared with hips with Tonnis $0: 15(55.6 \%)$ versus $10(12.7 \%)$, respectively, $p<0.001$. Men presented a higher prevalence

\section{Keywords}

- femoracetabular impingement

- arthroscopy

- hip of grades III and IV lesions than women, 23 (34.3\%) versus 2 (5.0\%), $p=0.001$, and had significantly higher functional scores $(65.6 \pm 19.6$ versus $49.3 \pm 21.6, p<0.001)$.

Conclusion Men presented a higher prevalence of deep lesions. Hips classified as Tonnis 1 presented a 4.4-fold higher probability of presenting these lesions. Patients with deep chondrolabral lesions had a better preoperative functional score.

\section{Introdução}

Desde o entendimento, por Beck et al, ${ }^{1}$ do mecanismo de lesão condral decorrente do impacto femoroacetabular (IFA) tipo CAME, no qual a delaminação condral ocorre pelas forças de cisalhamento provocadas pela alteração da morfologia normal da junção cabeça-colo do fêmur, inúmeros estudos têm sido realizados para potencializar o diagnóstico e o tratamento precoce dessas lesões, bem como avaliar resultados do tratamento cirúrgico, com técnicas como microfratura, implante de condrócitos, cola de fibrina, entre outros, que são dependentes da pobre capacidade de regeneração do tecido condral. ${ }^{2}$

É conhecimento corrente que pacientes com diagnóstico de osteoartrite do quadril apresentam risco elevado de conversão precoce em artroplastia ${ }^{3}$ e de falha do tratamento artroscópico, ${ }^{4}$ e que lesões condrais isoladas do quadril são fatores de mau prognóstico do tratamento cirúrgico. ${ }^{5-7} \mathrm{~A}$ delaminação condral ocorre frequentemente em pacientes com IFA..$^{1,8-11}$ Seu diagnóstico, entretanto, muitas vezes é feito somente durante o procedimento cirúrgico e, algumas vezes, pacientes com sintomatologia não exuberante apresentam extensas lesões cartilaginosas.

Já há consenso que alguns fatores estão associados à presença de lesões condrais. Além do achado pré-operatório dessas lesões em estudos de ressonância magnética (RM), ${ }^{12}$ a presença de artrose grau II ou superior de Tonnis, ${ }^{13}$ e o impacto tipo CAME tem associação direta com o desenvolvimento dos flaps condrais. ${ }^{14}$ Estudos também demonstram associação de lesões condrais no quadril com idade e sexo masculino. ${ }^{1,4,13}$

No diagnóstico transoperatório, são usadas classificações para determinar o comprometimento da cartilagem articular. A classificação clássica de Outerbridge, ${ }^{15}$ universal, que é usada para classificar lesões condrais em várias articulações, é moderadamente reproduzível e confiável. ${ }^{16}$ A classificação de Beck foi desenvolvida para a classificação de lesões condrais durante luxação controlada do quadril para tratamento do IFA. ${ }^{17}$ Mais recentemente, a classificação artroscópica de $\operatorname{Haddad}^{18}$ (-Tabela 1), descrita especificamente para lesões encontradas no quadril durante procedimento artroscópico e que faz relação com progressão da lesão condral. Essa classificação determina a localização da lesão em quadrantes, o dano à junção condrolabral e, por fim, a extensão da lesão. 0 dano à junção condrolabral é definido pela progressão da lesão; o grau 0 corresponde à ausência de lesão, o grau I à presença de wave sign ( - Fig. 1A), o grau II a dano à junção condrolabral, mas sem delaminação da cartilagem acetabular ( - Fig. 1B), e o grau III a quando há descolamento da cartilagem do osso subcondral (-Fig. 1C). Por fim, o grau IV corresponde à exposição do osso subcondral (-Fig. 1D). Essa classificação apresenta a melhor concordância intra- e interobservadores, quando comparada com as duas anteriores. ${ }^{17}$

Nenhuma manobra específica pode auxiliar no diagnóstico de lesões condrais isoladas do quadril, uma vez que a cartilagem articular não tem nocirreceptores e pode demandar tempo até que cause sintomas mecânicos ou de irritação articular. ${ }^{17} \mathrm{O}$ manejo precoce dessas lesões, associado à correção do IFA, tem relação direta com o resultado do

Tabela 1 Classificação artroscópica de Haddad ${ }^{18}$

\begin{tabular}{|l|l|}
\hline Grau 0 & Ausência de lesão condral. \\
\hline Grau I & $\begin{array}{l}\text { Wave sign, perda da fixação ao } \\
\text { osso subcondral. }\end{array}$ \\
\hline Grau II & $\begin{array}{l}\text { Óbvia ruptura da junção condrolabral, } \\
\text { mas na exploração com probe, } \\
\text { não há evidência de delaminação da } \\
\text { cartilagem do osso subcondral. }\end{array}$ \\
\hline Grau III & $\begin{array}{l}\text { Delaminação com separação da cartilagem } \\
\text { do osso subcondral. }\end{array}$ \\
\hline Grau IV & Osso subcondral exposto. \\
\hline
\end{tabular}




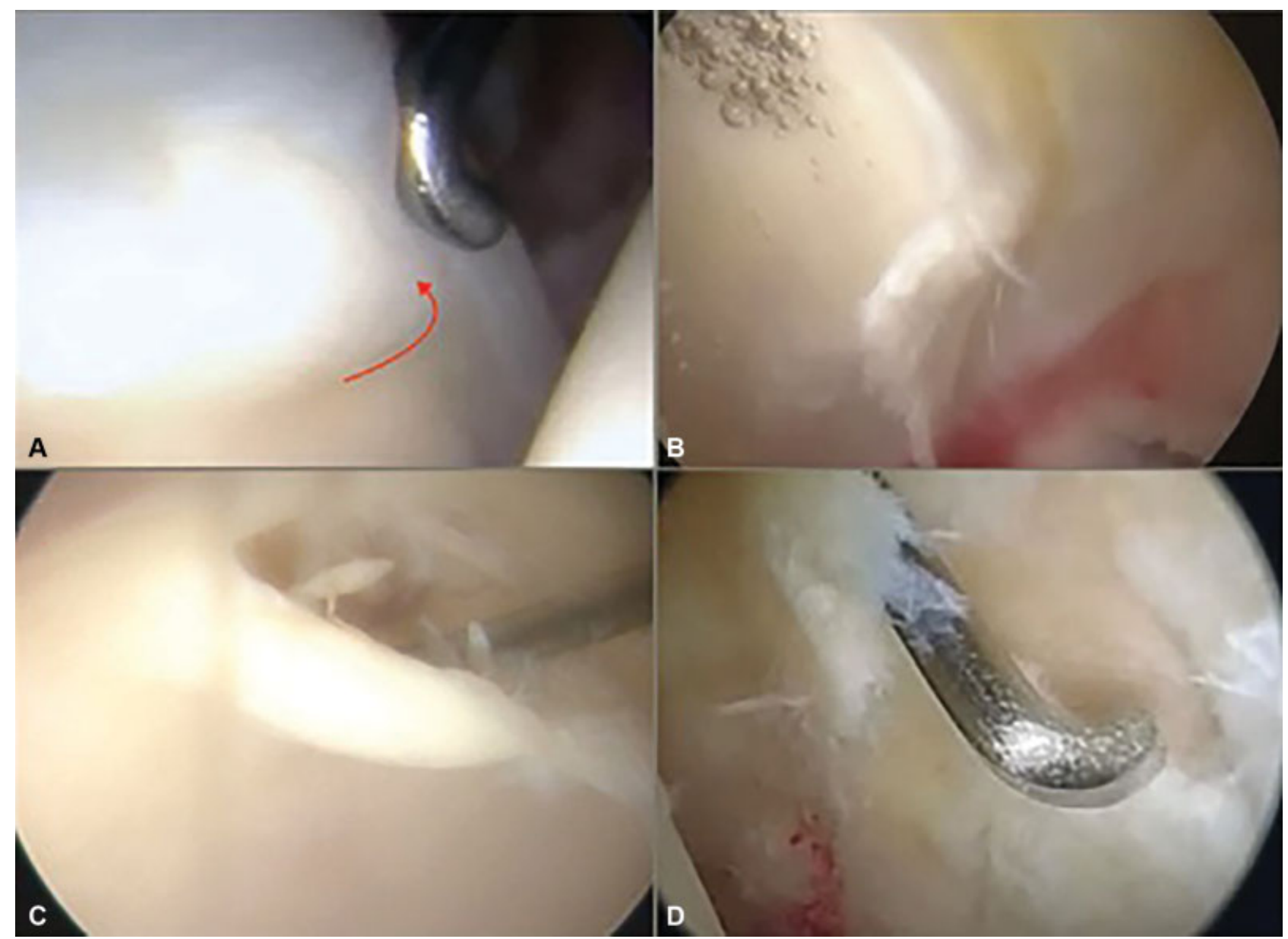

Fig. 1 (A) Grau I - wave sign; (B) Grau II - dano à junção condrolabral, sem delaminação da cartilagem; (C) Grau III - descolamento da cartilagem do osso subcondral; (D) Grau IV - exposição do osso subcondral.

tratamento artroscópico. ${ }^{19}$ Dessa forma, identificar pacientes suscetíveis a apresentar lesões profundas na cartilagem articular é decisivo para definir o momento do tratamento cirúrgico, bem como fazer o manejo das expectativas pósoperatórias do paciente, especialmente quanto ao retorno a atividades esportivas.

O objetivo do presente trabalho é avaliar se existe relação entre a presença de lesões condrais profundas no quadril durante tratamento artroscópico do IFA, relacioná-las ao escore NAHS-Brasil pré-operatório ${ }^{20}$ e a medidas pré-operatórias do ângulo alfa, ao ângulo de cobertura acetabular, à classificação radiológica de Tonnis, à idade e ao gênero dos pacientes.

\section{Material e Métodos}

O presente estudo foi aprovado pelo Comitê de Ética da Universidade de Passo Fundo, Passo Fundo, estado do Rio Grande do Sul, Brasil, sob o parecer número 1.749.565, conforme a Resolução 466/12 do Conselho Nacional de Saúde. $O$ presente estudo incluiu 110 pacientes (125 quadris) submetidos consecutivamente a artroscopia do quadril, entre maio de 2016 e maio de 2017, por um único cirurgião, que foram avaliados prospectivamente para a presença de lesões condrais em quadris. Foram excluídos da amostra inicial pacientes submetidos a artroscopia para tratamento de lesões diversas do IFA, com histórico de cirurgias prévias no quadril ou coluna, que apresentavam no momento da cirurgia artrose classificada pelas radiografias panorâmicas da pelve como artrose grau II de Tonnis e que não tiveram seus escores funcionais coletados no dia da cirurgia. Restou uma amostra de 92 pacientes (107 quadris), submetidos a tratamento do impacto misto (78\%) e a tratamento do impacto CAME isolado (22\%) e que apresentavam radiografias de pelve classificadas como 0 e 1 de Tonnis. Os pacientes foram submetidos a um questionário para avaliação funcional no dia da cirurgia e tiveram as medidas radiológicas e a classificação quanto ao grau de artrose pelos critérios de Tonnis. Foram usados para medida do ângulo de cobertura acetabular de Wiberg e para classificação radiológica de Tonnis para artrose do quadril a radiografia anteroposterior (AP) da pelve, com controle de rotação (sínfise púbica alinhada ao cóccix), e tilt (distância da sínfise púbica à junção sacrococcígea). Para medida do ângulo alfa, foram usadas incidências de Dunn de $45^{\circ}$. Os dados foram tabulados com o software Microsoft Excel (Microsoft Corporation, Redmond, WA, EUA), e a análise estatística foi feita com o IBM SPSS Statistics for Windows, Versão 22.0 (IBM Corp., Armonk, NY, EUA). Para a análise, as unidades amostrais consideradas foram os quadris, agrupados conforme a gravidade da lesão condrolabral em dois grupos, e quanto à profundidade da 
lesão condral, independentemente da localização e extensão da lesão, conforme a classificação artroscópica de Haddad, ${ }^{18}$ em um grupo sem lesão condral e com lesões consideradas superficiais, de graus I a II - e em outro com lesões profundas, de graus III e IV de Haddad. ${ }^{18}$ As associações entre o grau de lesão e o escore de sintomas, o ângulo de cobertura lateral, o ângulo alfa e a idade foram testadas com análise de variância (ANOVA, na sigla em inglês) com um critério de classificação. As associações entre o grau de lesão e gênero ou a classificação de Tonnis foram avaliadas com o teste de qui-quadrado. Consideraram-se como estatisticamente significativos os testes com valor de probabilidade $<0,05$.

\section{Resultados}

Foram consideradas 107 articulações, de 92 pacientes, das quais 67 (62,6\%) correspondiam a pacientes do sexo masculino. A idade média foi de 33,56 $\pm 7,4$ anos, e 59 (55,1\%) quadris eram esquerdos. Em relação ao grau de lesão, 11 quadris apresentavam lesões grau IV, e 14 quadris lesões grau III, totalizando 25 pacientes (23,4\%), agrupados como grupo de lesões profundas. No grupo de pacientes considerados como lesões leves, havia $13(12,1 \%)$ pacientes grau 0 (sem lesão condral), 24 (22,4\%) com lesões condrais grau I, e 45 (42,1\%) com lesões grau II, totalizando 82 quadris (76,6\%) nesse grupo. Em relação à presença de alterações radiográficas pelos critérios de Tonnis, foram 80 quadris $(74,8 \%$ ) que apresentavam quadris grau 0 , enquanto 27 (25,2\%) quadris apresentavam grau I de Tonnis.

Os homens submetidos a tratamento artroscópico do IFA apresentaram escores funcionais NAHS mais elevados e maior proporção de lesões condrais profundas do que os das pacientes do sexo feminino; $65,6 \pm 19,6$ versus $49,3 \pm 21,6 ; p<0,001$, e $23(34,3 \%)$ versus 2 (5,0\%); $p=0,001$, respectivamente.

Observou-se que os pacientes com lesões mais profundas apresentaram escores de avaliação NAHS significativamente maiores do que aqueles com lesões mais superficiais, 67,9 \pm 19,4 versus $57,0 \pm 21,9 ; p=0,027$. Não se observou diferença estatisticamente significativa entre os grupos com lesão superficial e profunda quanto ao ângulo de cobertura lateral de Wiberg e ao ângulo alfa, $35,1 \pm 8,4$ versus $33,1 \pm 6,6 ; p=$ 0,271 ; e $66,3 \pm 8,5$ versus $69,0 \pm 7,0 ; p=0,155$, respectivamente. Não se observou diferença estatisticamente significativa quanto à idade dos pacientes correspondentes a lesões condrais superficiais ou profundas, 33,0 \pm 7,6 versus $35,0 \pm$ 7,$4 ; p=0,253$. Por outro lado, a proporção de Tonnis 1 foi significativamente maior entre as lesões condrais mais profundas, $15(60,0 \%)$, do que entre as mais superficiais, 12 (14,6\%); $p<0,001$. A prevalência de lesões condrais profundas foi maior entre os quadris classificados como Tonnis $1 \mathrm{em}$ relação aos quadris Tonnis 0: 15 (55,6\%) versus 10 (12,7\%), razão de prevalência 4,4; $p<0,001$ (- Tabela 2 ).

\section{Discussão}

A presença de lesões condrais em pacientes submetidos a artroscopia do quadril para tratamento do IFA é um achado frequente, e é documentada entre 45 e $75 \%$ das cirurgias para
Tabela 2 Grau de lesão e variáveis analisadas

\begin{tabular}{|l|l|l|l|}
\hline & $\begin{array}{l}\text { Lesão ausente } \\
\text { ou leve }(\boldsymbol{n}=\mathbf{8 2})\end{array}$ & $\begin{array}{l}\text { Lesão } \\
\text { profunda } \\
(\boldsymbol{n}=\mathbf{2 5})\end{array}$ & valor-p \\
\hline $\begin{array}{l}\text { Gênero } \\
\text { (masculino) }\end{array}$ & $44(65,7 \%)$ & $23(34,3 \%)$ & 0,001 \\
\hline Idade (anos) & $33,0 \pm 7,6$ & $35,0 \pm 7,4$ & 0,253 \\
\hline NAHS & $57,0 \pm 21,9$ & $67,9 \pm 19,4$ & 0,027 \\
\hline Ângulo alfa & $66,3 \pm 8,5$ & $69,0 \pm 7,0$ & 0,158 \\
\hline Ângulo CE & $35,1 \pm 8,4$ & $33,1 \pm 6,6$ & 0,288 \\
\hline Tonnis I & $10(12,7 \%)$ & $15(55,6 \%)$ & $<0,001$ \\
\hline
\end{tabular}

Abreviações: CE, Ângulo da borda central; NAHS, escore de quadril não artrítico.

Valores expressam média \pm desvio padrão ou frequência absoluta e relativa.

tratamento do impacto femoroacetabular. ${ }^{9,21,22}$ Em nossa série, a incidência foi de $87,9 \%$. Bhatia et al $^{19}$ apresentaram prevalência de $28 \%$ de lesões grau IV de Outerbridge em pacientes submetidos a artroscopia do quadril, das quais $78 \%$ eram lesões isoladas no acetábulo. Foram fatores de risco independentes a presença de redução do espaço articular e a longa duração dos sintomas. Em nosso estudo, encontramos lesões condrais profundas (graus III e IV de Haddad ${ }^{18}$ ) em $23,4 \%$ dos pacientes. Entretanto, essas lesões não significam presença de processo degenerativo (artrose), já que as lesões condrais classsificadas como profundas foram provocadas por ruptura da junção condrolabral seguida de destacamento da cartilagem acetabular do osso subcondral. São, portanto, delimitadas e não relacionadas a processo degenerativo difuso articular. Essas lesões condrais são candidatas ao tratamento artroscópico. ${ }^{23}$

As lesões agrupadas como profundas no presente estudo podem ser classificadas como Outerbridge IV, já que expõem o osso subcondral, como grau III por terem descolamento macroscópico da cartilagem do osso subcondral, e como grau IV por apresentarem exposição do osso acetabular. No presente estudo, não encontramos uma relação estatisticamente significativa entre as medidas do ângulo alfa e a presença dessas lesões, em contraste com os achados de Bhatia et al, ${ }^{19}$ que demonstraram que grandes ângulos alfa estão relacionados à presença de lesões condrais grau IV de Outerbridge.

Claßen et al, ${ }^{24}$ em um estudo com 177 pacientes submetidos a artroscopia do quadril para tratamento do impacto tipo CAME isolado, concluíram que a análise do NAHS também é fator preditivo de lesões condrais, entretanto pouco confiável, com baixa sensibilidade e especificidade, de $62,8 \%$ e $68,7 \%$, respectivamente, quando usado um ponto de corte de 42,5 pontos, com definição de presença de lesões condrais pela classificação de Outerbridge. Quando o ponto de corte é definido como 30 pontos do NAHS, a especificidade é de $100 \%$, e a sensibilidade de $1 \%$. Nossa análise, a qual avaliou pacientes submetidos à artroscopia do quadril para tratamento de impactos dos tipos CAME e misto, demonstrou relação inversa de gravidade das lesões com escore funcional não artrítico, já que o grupo de pacientes com lesões condrais 
consideradas profundas apresentou melhores escores funcionais pré-operatórios. Surpreende esse resultado, o qual sugere que os pacientes com melhores escores funcionais têm associadas piores lesões articulares. Há poucos estudos que avaliam o tratamento conservador do IFA. Wall et al, ${ }^{25} \mathrm{em}$ uma revisão sistemática da literatura, concluíram que há indícios de que fisioterapia e modificação do estilo de vida proporcionam algum benefício ao paciente. Emara et al, ${ }^{26} \mathrm{em}$ uma análise prospectiva de série de casos com 37 pacientes, demonstraram melhoria do NAHS médio de 72 para 91 pontos após tratamento conservador, que incluiu restrição de sobrecarga articular, uso de anti-inflamatórios, fisioterapia, e modificação de atividades que predispõem ao IFA, com seguimento médio de 25 a 28 meses, e que resultou em 4 falhas que necessitaram de cirurgia. Uma vez que há relação entre a duração dos sintomas com a incidência de lesões condrais, ${ }^{19,25}$ suspeitamos que pacientes que apresentam dor crônica possam modificar seu estilo de vida e atividades de sobrecarga do quadril, o que melhora parcialmente o escore funcional.

$\mathrm{Na}$ amostra estudada, não houve diferença significativa $(p=0,253)$ entre idade e lesões profundas, embora a média de idade seja discretamente maior nos pacientes como lesões condrais consideradas profundas. Homens apresentaram melhores escores funcionais e também maior prevalência de lesões profundas, achado compatível com estudos anteriores. ${ }^{1,4,13}$

A principal limitação do presente estudo foi não levar em consideração o tempo pelo qual o paciente foi exposto à lesão condral, de modo que não foi possível estimar o impacto que o tempo de lesão tem no desfecho (quantidade de sintomas medidos pelo escore). Além disso, a amostra é predominantemente de pacientes submetidos a tratamento do IFA misto, e há indícios na literatura de que o impacto pincer seja fator protetor para delaminação condral. ${ }^{14}$

\section{Conclusão}

Em nossa amostra, pacientes com lesões condrais profundas no quadril apresentaram escores NAHS significativamente melhores do que pacientes sem lesão condral ou com lesões consideradas leves. Quadris classificados como Tonnis 1 tiveram probabilidade 4,4 vezes maior de apresentar lesões condrais profundas quando comparados com os de pacientes classificados como Tonnis 0 . Homens submetidos a tratamento do IFA apresentaram melhores escores funcionais pré-operatórios do que as mulheres e, ainda, maior prevalência de lesões graus III e IV de Haddad ${ }^{18}$. Não houve associação estatisticamente significativa da presença de lesões condrais profundas com ângulo alfa, ângulo de cobertura lateral e idade.

\section{Conflitos de Interesses}

Os autores declaram não haver conflitos de interesses.

\section{Referências}

1 Beck M, Kalhor M, Leunig M, Ganz R. Hip morphology influences the pattern of damage to the acetabular cartilage: femoroacetabular impingement as a cause of early osteoarthritis of the hip. J Bone Joint Surg Br 2005;87(07):1012-1018
2 Zamber RW, Teitz CC, McGuire DA, Frost JD, Hermanson BK. Articular cartilage lesions of the knee. Arthroscopy 1989;5(04): 258-268

3 Schairer WW, Nwachukwu BU, McCormick F, Lyman S, Mayman D. Use of hip arthroscopy and risk of conversion to total hip arthroplasty: a population-based analysis. Arthroscopy 2016;32(04):587-593

4 Philippon MJ, Briggs KK, Yen YM, Kuppersmith DA. Outcomes following hip arthroscopy for femoroacetabular impingement with associated chondrolabral dysfunction: minimum two-year follow-up. J Bone Joint Surg Br 2009;91(01):16-23

5 Egerton T, Hinman RS, Takla A, Bennell KL, O'Donnell J. Intraoperative cartilage degeneration predicts outcome 12 months after hip arthroscopy. Clin Orthop Relat Res 2013;471(02):593-599

6 Haviv B, O'Donnell J. Arthroscopic treatment for acetabular labral tears of the hip without bony dysmorphism. Am J Sports Med 2011;39(39, Suppl)79S-84S

7 Sankar WN, Matheney TH, Zaltz I. Femoroacetabular impingement: current concepts and controversies. Orthop Clin North Am 2013;44(04):575-589

8 Peters CL, Erickson J. The etiology and treatment of hip pain in the young adult. J Bone Joint Surg Am 2006;88(Suppl 4):20-26

9 Beck M, Leunig M, Parvizi J, Boutier V, Wyss D, Ganz R. Anterior femoroacetabular impingement: part II. Midterm results of surgical treatment. Clin Orthop Relat Res 2004;(418):67-73

10 Beaulé PE, Zaragoza E, Copelan N. Magnetic resonance imaging with gadolinium arthrography to assess acetabular cartilage delamination. A report of four cases. J Bone Joint Surg Am 2004;86(10):2294-2298

11 Beaulé PE, Zaragoza EJ. Surgical images: musculoskeletal acetabular cartilage delamination demonstrated by magnetic resonance arthrography: inverted "Oreo" cookie sign. Can J Surg 2003; 46(06):463-464

12 Ho CP, Ommen ND, Bhatia S, et al. Predictive value of 3-T magnetic resonance imaging in diagnosing grade III and IV chondral lesions in the hip. Arthroscopy 2016;32(09):1808-1813

13 Chandrasekaran S, Darwish N, Gui C, Lodhia P, Suarez-Ahedo C, Domb BG. Outcomes of Hip Arthroscopy in Patients with Tönnis Grade-2 Osteoarthritis at a Mean 2-Year Follow-up: Evaluation Using a Matched-Pair Analysis with Tönnis Grade-0 and Grade-1 Cohorts. J Bone Joint Surg Am 2016;98(12):973-982

14 Anderson LA, Peters CL, Park BB, Stoddard GJ, Erickson JA, Crim JR. Acetabular cartilage delamination in femoroacetabular impingement. Risk factors and magnetic resonance imaging diagnosis. J Bone Joint Surg Am 2009;91(02):305-313

15 Outerbridge RE. The etiology of chondromalacia patellae. J Bone Joint Surg Br 1961;43-B:752-757

16 MacDonald AE, Bedi A, Horner NS, et al. Indications and outcomes for microfracture as an adjunct to hip arthroscopy for treatment of chondral defects in patients with femoroacetabular impingement: a systematic review. Arthroscopy 2016;32(01): 190-200.e2

17 Amenabar T, Piriz J, Mella C, Hetaimish BM, O’Donnell J. Reliability of 3 different arthroscopic classifications for chondral damage of the acetabulum. Arthroscopy 2015;31(08):1492-1496

18 Konan S, Rayan F, Meermans G, Witt J, Haddad FS. Validation of the classification system for acetabular chondral lesions identified at arthroscopy in patients with femoroacetabular impingement. J Bone Joint Surg Br 2011;93(03):332-336

19 Bhatia S, Nowak DD, Briggs KK, Patterson DC, Philippon MJ. Outerbridge grade IV cartilage lesions in the hip identified at arthroscopy. Arthroscopy 2016;32(05):814-819

20 Del Castillo LN, Leporace G, Cardinot TM, Levy RAL, Oliveira LP. Translation, cross-cultural adaptation and validation of the Brazilian version of the Nonarthritic Hip Score. Sao Paulo Med J 2013; 131(04):244-251

21 Beaulé PE, Le Duff MJ, Zaragoza E. Quality of life following femoral head-neck osteochondroplasty for femoroacetabular impingement. J Bone Joint Surg Am 2007;89(04):773-779 
22 Nepple JJ, Carlisle JC, Nunley RM, Clohisy JC. Clinical and radiographic predictors of intra-articular hip disease in arthroscopy. Am J Sports Med 2011;39(02):296-303

23 Mella C, Villalón IE, Núñez Á, Paccot D, Díaz-Ledezma C. Hip arthroscopy and osteoarthritis: Where are the limits and indications? SICOT J 2015;1:27

24 Claßen T, Körsmeier K, Kamminga M, et al. Is early treatment of cam-type femoroacetabular impingement the key to avoiding associated full thickness isolated chondral defects? Knee Surg Sports Traumatol Arthrosc 2016;24(07):2332-2337

25 Wall PD, Fernandez M, Griffin DR, Foster NE. Nonoperative treatment for femoroacetabular impingement: a systematic review of the literature. PM R 2013;5(05):418-426

26 Emara K, Samir W, Motasem H, Ghafar KA. Conservative treatment for mild femoroacetabular impingement. J Orthop Surg (Hong Kong) 2011;19(01):41-45 